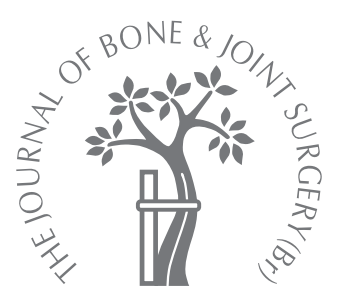

I. Nagura, H. Fujioka, T. Kokubu, T. Makino, Y. Sumi, M. Kurosaka

From Kobe University Graduate School of Medicine, Kobe, Japan

I. I. Nagura, MD, Orthopaedic Surgeon

H. Fujioka, MD, Lecturer

II T. Kokubu, MD, Orthopaedic Surgeon

- T. Makino, MD, Research

Associate

M. Kurosaka, MD, Professor and Chairman

Department of Orthopaedic Surgery

Kobe University Graduate School of Medicine, 7-5-1 Kusunoki-cho, Chuo-ku, Kobe 650-0017, Japan.

I. Sumi, PhD, Group Leader, Senior Manager

Department of Tissue

Engineering Development,

Innovation Research Institute TEIJIN Limited, 4-3-2

Asahigaoka, Hino, Tokyo 1918512, Japan.

Correspondence should be sent to $\mathrm{Dr}$ H. Fujioka; e-mail: hfujioka@med.kobe-u.ac.jp

(C)2007 British Editorial Society of Bone and Joint Surgery doi:10.1302/0301-620X.89B2. $17754 \$ 2.00$

$J$ Bone Joint Surg $[\mathrm{Br}]$ 2007;89-B:258-64.

Received 10 February 2006; Accepted after revision 16 August 2006

\title{
Repair of osteochondral defects with a new porous synthetic polymer scaffold
}

\begin{abstract}
We developed a new porous scaffold made from a synthetic polymer, poly(DL-lactide-coglycolide) (PLG), and evaluated its use in the repair of cartilage. Osteochondral defects made on the femoral trochlear of rabbits were treated by transplantation of the PLG scaffold, examined histologically and compared with an untreated control group.

Fibrous tissue was initially organised in an arcade array with poor cellularity at the articular surface of the scaffold. The tissue regenerated to cartilage at the articular surface. In the subchondral area, new bone formed and the scaffold was absorbed. The histological scores were significantly higher in the defects treated by the scaffold than in the control group ( $\mathrm{p}<0.05)$.

Our findings suggest that in an animal model the new porous PLG scaffold is effective for repairing full-thickness osteochondral defects without cultured cells and growth factors.
\end{abstract}

The low mitotic activity of chondrocytes causes problems in the use of chondral tissue for the repair of osteochondral lesions. ${ }^{1}$ In order to treat focal chondral lesions, techniques such as abrasion arthroplasty, subchondral drilling, autologous osteochondral grafting, periosteal-perichondral grafting and chondrocyte transplantation have been developed and have achieved acceptable results. ${ }^{2}$ However, several problems still remain. Articular cartilage regenerated by abrasion arthroplasty is not hyaline cartilage but fibrous cartilage. ${ }^{3}$ Autologous osteochondral cylindrical grafting can be used to repair osteochondral defects, but there is a limit to the size and shape of the cartilage lesion which can be successfully treated. ${ }^{4}$ Morbidity and pain at the donor site are also a consideration. Furthermore, transplantation of cultured chondrocytes requires a number of surgical procedures. ${ }^{5}$

Previous studies have shown that small fullthickness cartilage defects can be regenerated by erupted bone-marrow cells containing osteoprogenitor cells. ${ }^{1,6}$ In rabbits, untreated full-thickness osteochondral defects, $3 \mathrm{~mm}$ in diameter, have been shown to heal spontaneously. ${ }^{6}$ During the repair process, fibrous tissues which had been organised into an arcade array with mesenchymal cells at the articular surface developed into cartilage tissue. This was followed by osteoblastic synthesis of woven bone in the depth of the defect. It was speculated that in small osteochondral defects, erupted bone marrow cells filled the defect quickly, improving the irregularity of the articular surface and subsequently regenerated cartilage at the surface and bone at the depth of the defect. ${ }^{6}$

In contrast, when full-thickness osteochondral defects, $6 \mathrm{~mm}$ in diameter and in depth, were made in goat femoral condyles, bonemarrow cells containing osteoprogenitor cells erupted from the bottom of the defect, but failed to fill it. ${ }^{7}$ In these larger defects erupted bone-marrow cells did not regenerate either bone at the deep zone, or cartilage at the surface, resulting in resorption of the osseous wall of the defect, the formation of large cavities, and the collapse of the surrounding articular cartilage and subchondral bone. ${ }^{7}$ It was speculated that an osteochondral defect of a critical size could not be filled with reparative tissue and could progress to the creation of a large defect in the subchondral bone.

We hypothesised that an osteochondral defect $5 \mathrm{~mm}$ in diameter could be repaired with biological matrices which prevent the collapse of the surrounding articular cartilage and subchondral bone and hold the erupted bone-marrow cells within the defect. We developed a bio-absorbable scaffold made from synthetic polymer. It had multiple pores, into which the erupted bone-marrow cells could infiltrate and attach. In order to analyse the efficacy of cartilage repair with this new porous scaffold, we transplanted it into osteochondral defects 


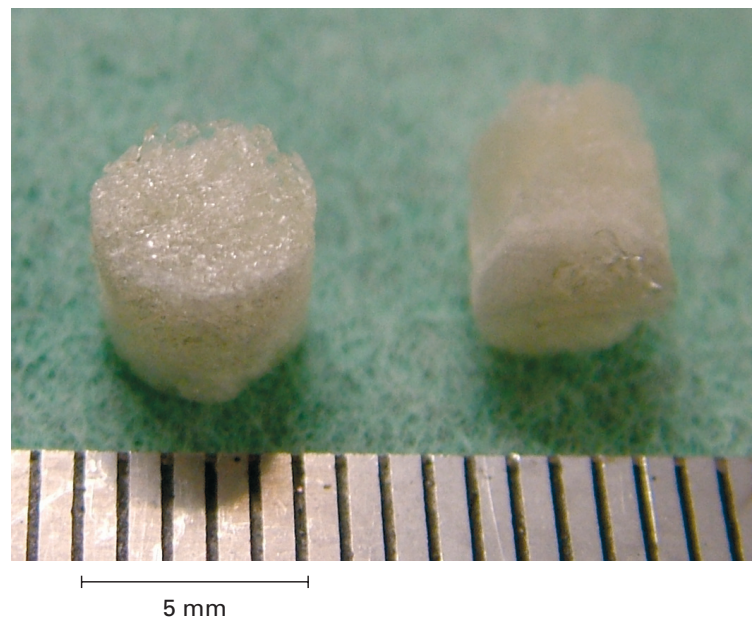

Fig. 1a

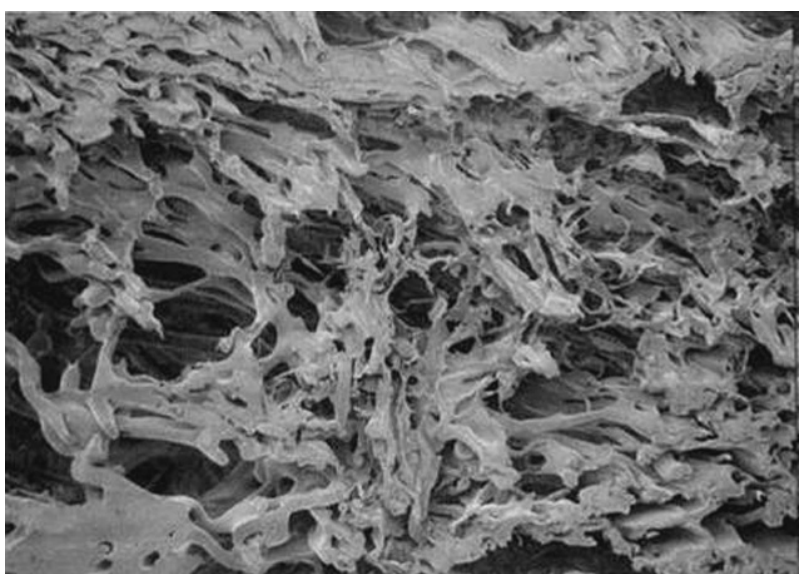

$100 \mu \mathrm{m}$

Fig. 1b

a) photograph of the new porous poly (DL-lactide-co-glycolide) (PLG) scaffold and b) a photomicrograph of the cylindrical porous PLG scaffold.

made on the femoral trochlea of rabbits and performed macroscopic and histological examinations.

\section{Material and Methods}

Development of the scaffold. We purchased poly (DLlactide-co-glycolide) (PLG) from Absorbable Polymers International Inc (Pelham, Alabama). The molar ratio of lactide to glycolide was 50:50 and the inherent viscosity was $1.08 \mathrm{dl} / \mathrm{g}$ measured at $30^{\circ} \mathrm{C}$ in hexafluoroisopropanol. Dimethylsulphoxide (DMSO) was purchased from WAKO Pure Chemical Industries Ltd (Osaka, Japan). PLG and DMSO were used without further purification. The PLG was dissolved in DMSO to give $10 \%$ (weight by volume) polymer dope which was pored into a container, frozen at the rate of $1^{\circ} \mathrm{C} / \mathrm{min}$ temperature decrease, and freeze-dried in vacuo. Lyophilised PLG sponge was soaked in 70/30 (volume by volume) of ethanol-water mixed solution and washed by the same solution to extract residual DMSO, then dried under reduced pressure. Thereafter, the purified PLG scaffold was obtained (Fig. 1a).

The pore size of the PLG scaffold was confirmed by a scanning electron microscope (JSM-5310; JEOL Ltd, Tokyo, Japan) at an accelerated voltage of $2 \mathrm{kV}$ and a tilt angle of $30^{\circ}$. The sizes of most pores were less than $100 \mu \mathrm{m}$ (Fig. 1b).

The total porosity $(\Pi)$ of the PLG scaffold was measured by gravimetry according to the equation:

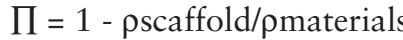

where $\rho_{\text {materials }}$ was the density of the material of which the scaffold was made and $\rho_{\text {scaffold }}$ was the apparent density of the scaffold measured by dividing the weight by the volume of the scaffold. The mean total porosity of the PLG scaffold was 0.83 (0.80 to 0.85$)$.
Operative procedures. All the experiments were reviewed and approved by the Animal Research Committee of Kobe University Graduate School of Medicine. We used 60 skeletally-mature female Japanese white rabbits (Kitayama LABES, Nagano, Japan) with a mean weight of $3.3 \mathrm{~kg}(2.8$ to 3.7$)$. All the operations were performed on the right knee of the rabbits under general anaesthesia using an intravenous pentobarbital sodium solution $(30 \mathrm{mg} / \mathrm{kg}$ bodyweight). In each rabbit, the right limb was disinfected and $3 \mathrm{ml}$ of $1 \%$ lidocaine was injected subcutaneously into the medial parapatellar region where the skin incision was to be made. A medial parapatellar approach was used to expose the knee. The femoral trochlea, which contacts the patella when the knee is flexed at $90^{\circ}$, was selected as the site for the osteochondral defect. A full-thickness cylindrical osteochondral fragment, $5 \mathrm{~mm}$ in diameter and $5 \mathrm{~mm}$ in depth, was removed using the Osteochondral Autograft Transfer System (OATS; Arthrex, Naples, Florida). After irrigating the joint with sterile isotonic saline, the rabbits were randomised into two groups of 30. In the PLG group, the defect was repaired by transplantation of the PLG scaffold, while in the control group it was untreated. The joint capsule and skin were sutured as separate layers. After surgery, all the rabbits were returned to their cages and allowed to move freely without immobilisation of the joint.

At 1, 3, 6, 12 and 24 weeks after the operation, six rabbits from each group were killed by an intravenous injection of pentobarbital sodium and the femoral trochlea of the right knee was taken and examined macroscopically and histologically. Additionally, in order to confirm the expression of type-II collagen in the tissue regenerated by the PLG scaffold, specimens of the PLG group at week 24 were examined immunohistochemically using an anti-type II collagen antibody. 
Table I. Histological grading scale modified from that of Wakitani et $\mathrm{al}^{8}$

\begin{tabular}{|c|c|c|}
\hline & Category & Points \\
\hline \multirow[t]{6}{*}{$\bar{A}$} & Cell morphology & \\
\hline & Hyaline cartilage & 4 \\
\hline & Mostly hyaline cartilage & 3 \\
\hline & Mostly fibrocartilage & 2 \\
\hline & Mostly non-cartilage & 1 \\
\hline & Non-cartilage only & 0 \\
\hline \multirow[t]{5}{*}{ B } & Matrix staining (metachromasia) & \\
\hline & Normal (compared with host) & 3 \\
\hline & Slightly reduced & 2 \\
\hline & Significantly reduced & 1 \\
\hline & No staining & 0 \\
\hline \multirow[t]{5}{*}{ C } & Surface regularity ${ }^{*}$ & \\
\hline & Smooth $(>3 / 4)$ & 3 \\
\hline & Moderate $(1 / 2$ to $3 / 4)$ & 2 \\
\hline & Irregular $(1 / 4$ to $1 / 2)$ & 1 \\
\hline & Severely irregular $(<1 / 4)$ & 0 \\
\hline \multirow[t]{4}{*}{ D } & Thickness of cartilage $^{\dagger}$ & \\
\hline & $>2 / 3$ & 2 \\
\hline & $1 / 3$ to $2 / 3$ & 1 \\
\hline & $<1 / 3$ & 0 \\
\hline \multirow[t]{4}{*}{$E$} & Integration of donor to host adjacent cartilage & \\
\hline & Both edges integrated & 2 \\
\hline & One edge integrated & 1 \\
\hline & Both edges not integrated & 0 \\
\hline
\end{tabular}

Histological analysis. The specimens were fixed in $4 \%$ paraformaldehyde for 24 hours, decalcified with $0.25 \mathrm{~mol} /$ 1 EDTA in phosphate-buffered saline at $\mathrm{pH} 7.5$, dehydrated in graded alcohol solutions, and embedded in paraffin wax. Sagittal sections ( $7 \mu \mathrm{m}$ thick) were cut and stained with haematoxylin and eosin or Toluidine Blue, and examined by light microscopy. The histological findings were scored according to a histological grading scale modified from that described by Wakitani et al, ${ }^{8}$ so that higher quality of cartilage repair led to higher scores. This scale contained five categories with a high score of 14 points (Table I). Each section was scored randomly by three blinded individuals (IN, TK, TM). Statistical significance was performed using Welchs's $t$-test and p-values $\leq 0.05$ were considered to be statistically significant.

Immunohistochemical analysis. Deparaffinised sections were digested with proteinase (Dako Cytomation Inc., Via Real Carpinteria, California) for ten minutes and treated with $3 \%$ hydrogen peroxide (WAKO Pure Chemical Industries Ltd) to block the activity of endogenous peroxidase. The sections were treated in a 1:50 dilution of goat anti-type II collagen antibodies (Southern Biotechnology Associates, Birmingham, Alabama) at $4^{\circ} \mathrm{C}$ overnight and subsequently treated with peroxidase-labelled anti-goat immunoglobulin (Histofine Simplestain max $\mathrm{PO}(\mathrm{G})$; Nichirei Bioscience, Tokyo, Japan) at room temperature for 30 minutes. The signal was developed as a brown reaction product using peroxidase substrate 3,3-diaminobenzide (Histofine Simplestain DAB Solution; Nichirei Bioscience, Tokyo, Japan) and the sections examined microscopically.

\section{Results}

\section{Macroscopic findings}

No rabbits developed joint contracture or infection. No inflammatory reaction was observed in any specimen throughout the post-operative period. We examined the regularity of the cartilage surface compared with the surrounding cartilage.

PLG group. At post-operative week 1 the surface of the defect was covered with reddish granular tissue (Fig. 2a). At weeks 3 and 6, white reparative tissue was observed at the margin of the defect, but the central area of the defect was still depressed (Figs 2 b and 2c). At week 12, the surface of the defect was covered with white shiny reparative tissue (Fig. 2d) and at week 24, a smooth continuity of the articular surface between the operated region and the surrounding intact trochlea was observed (Fig. 2e).

Control group. The defect was concave and the margin of the defect was clearly recognised in all specimens obtained at post-operative weeks 1,3 and 6 (Figs $2 \mathrm{f}$ to $2 \mathrm{~h}$ ). At weeks 12 and 24, the defect was filled with newly-formed tissue, but the irregularity of the articular surface of the defect and the margin of the defect remained visible (Figs $2 \mathrm{i}$ and $2 \mathrm{j}$ ).

\section{Histological findings}

PLG group. At post-operative week 1, the PLG scaffold was not absorbed and fibrous tissue was observed at the articular surface of the scaffold (Fig. 3a). At a higher magnification $(\times 200)$, numerous blood cells were seen in the pores of the scaffold. Fibrous tissue was attached to the PLG scaffold at the articular surface and was organised in an arcade array with poor cellularity (Fig. 3b).

At week 3, remnants of the PLG scaffold in the defect had decreased and the formation of new bone was observed at the subchondral zone (Fig. 3c). Regenerated fibrous tissue with metachromatic matrix increased at the articular surface zone. At a higher magnification $(\times 200)$, proliferated cells were observed at the articular surface of the PLG scaffold (Fig. 3d).

At week 6, most of the PLG scaffold had been absorbed and replaced by newly-formed bone at the subchondral zone. Also, reparative tissue with metachromatic matrix had been regenerated at the articular surface of the PLG scaffold (Fig. 3e). At a higher magnification $(\times 200)$, roundshaped chondrocytes were observed at the superficial zone of the cartilaginous tissue and hypertrophic chondrocytes with a vacuole at the deeper zone of the cartilaginous tissue (Fig. 3f). At the subchondral area, dense and highlyvascularised newly-formed bone was observed.

At week 12, the thickness of regenerated cartilaginous tissue with metachromatic matrix at the articular surface and formation of bone at the subchondral zone had increased compared with those seen at post-operative week 


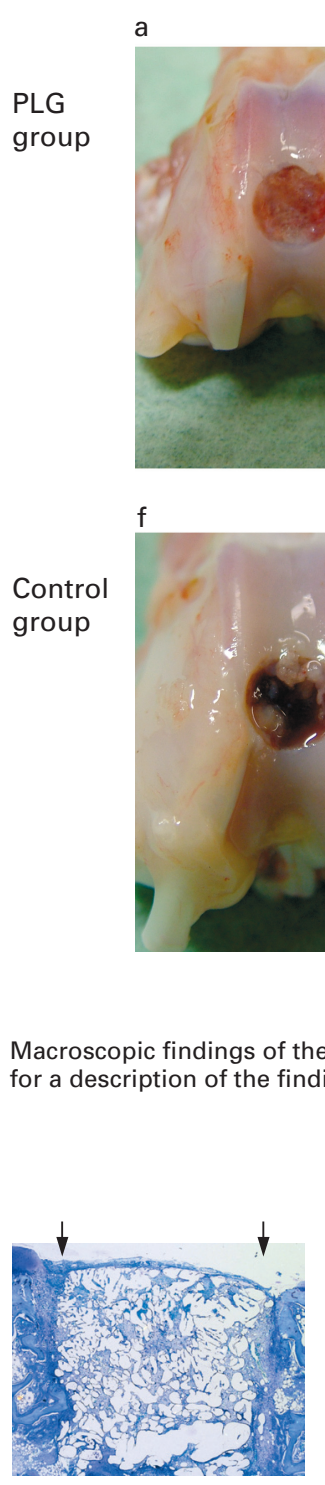

Fig. 3a Macroscopic findings of the troc

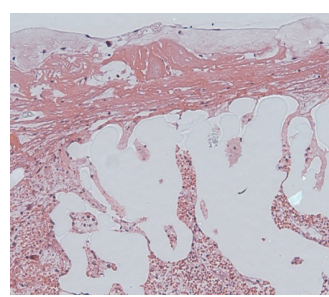

Fig. 3b b

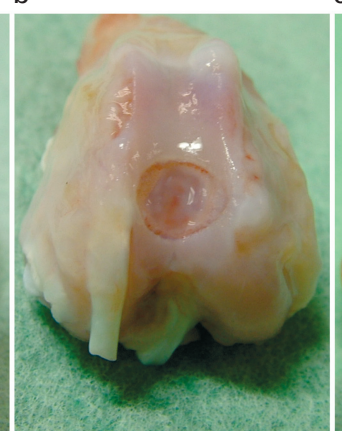

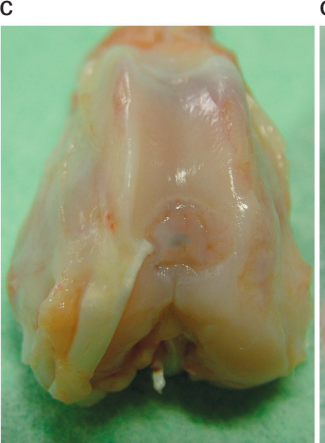

d

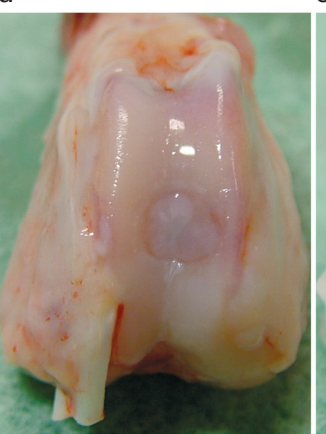

e

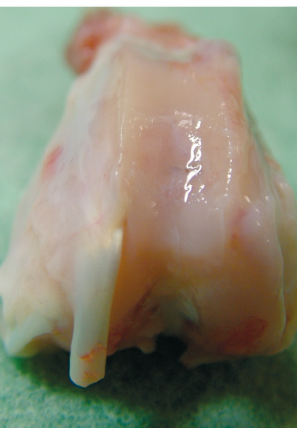

g
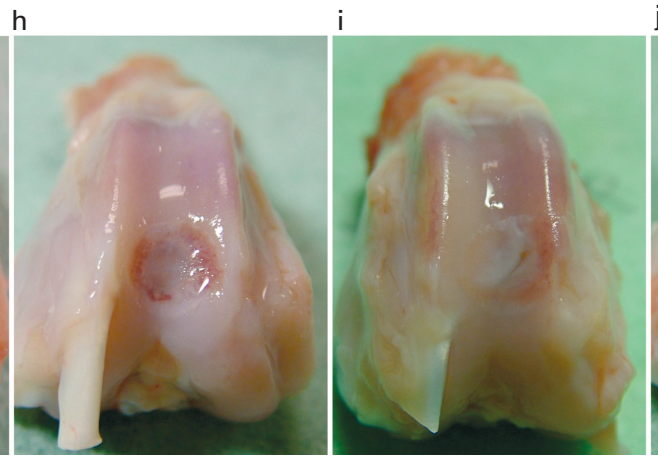

j

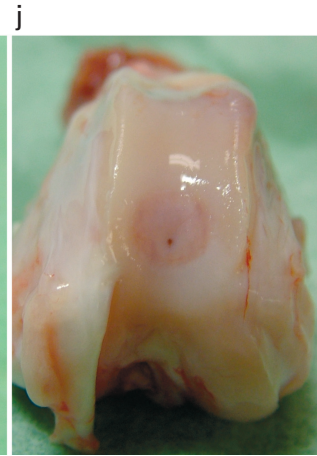

Fig. 2
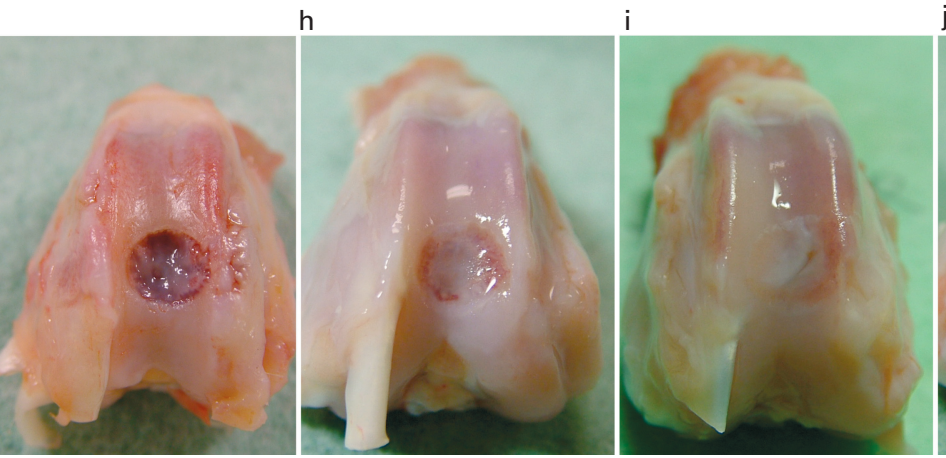

$3(b, g), 6(c, h), 12$ (d, i) and 24 (e, j) (PLG, poly (D-lactide-co-glycolide). See text

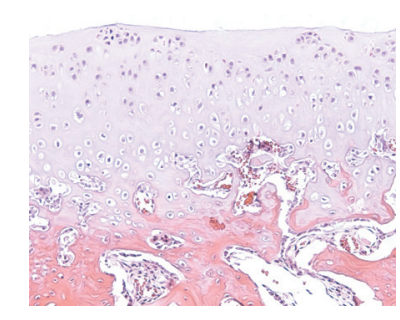

Fig. $3 f$

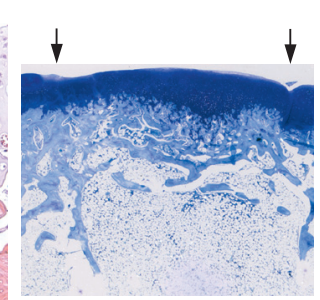

Fig. $3 g$

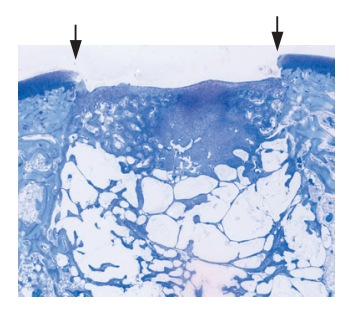

Fig. 3c

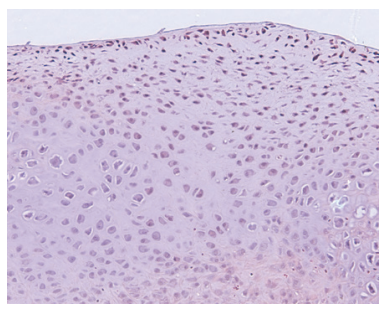

Fig. 3d

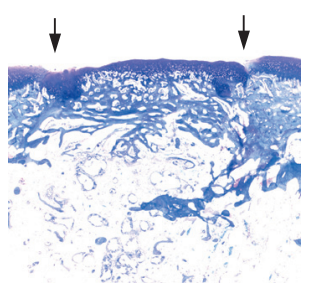

Fig. 3e

Photomicrographs showing the histological findings for the PLG (poly) DL-lactide-co-glycolide)) group.

6 (Fig. 3g). At a higher magnification $(\times 200)$, the chondrocytes had been replaced by dense and highly-vascularised new bone at the subchondral area (Fig. 3h).
At week 24, the thickness of the regenerated cartilage ayer and the pattern of bone trabeculae at the subchondral zone were the same as those at the adjacent normal articular cartilage (Fig. 3i). At a higher magnification $(\times 200)$, 


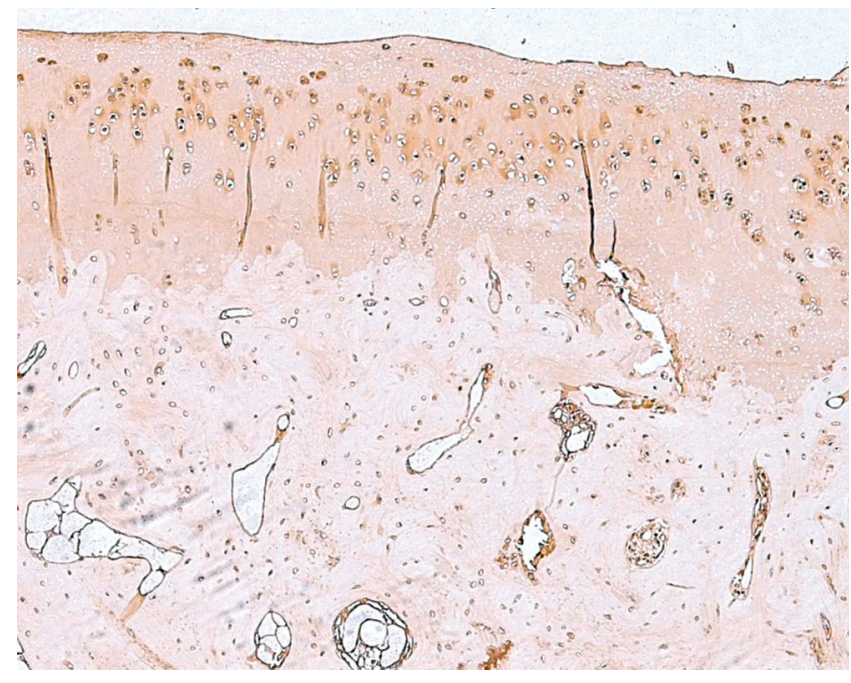

Fig. 4

Photomicrograph showing the immunohistochemical findings in the PLG (poly (DL-lactide-co-glycolide)) group. Type-II collagen was observed in regenerated cartilage at post-operative week $24(\times 60)$.

round-shaped chondrocytes at the superficial zone, hypertrophic chondrocytes with a vacuole at the middle, noncalcified zone, land calcified zones were observed and a tidemark between the calcified and the non-calcified zones was detected (Fig. 3j).

In the immunohistochemical analysis, type-II collagen was detected in the regenerated cartilage in the PLG group (see Fig. 4).

Control group. At post-operative week 1, the defect was filled with fibrous tissue (Fig. 5a) and at week 3, formation of new bone at the deep zone of the defect was seen (Fig. 5b). At week 6, reparative tissue with a metachromatic matrix at the articular surface and formation of bone at the subchondral area were observed (Fig. 5c). At week 12 , an increase in the reparative tissue with a metachromatic matrix and formation of bone at the deep zone of the defect were observed, but, a cleft was observed at the centre of the defect in most cases (Fig. 5d). At week 24, reparative cartilage and bone were detected at the rim of the osteochondral defect, but the centre of the osteochondral defect was not repaired and remained as a defect (Fig. 5e). At a higher magnification (¥ 200), the regenerated cartilage was thin and the arrangement of chondrocytes sparse, compared with those of the PLG group at week 24 (Fig. 5f).

Histological scores. At post-operative weeks 3, 6, 12 and 24, the histological findings were evaluated by a histological grading scale (Table I). The mean scores at each post-operative time point in the PLG and control groups are given in Table II and the total scores are summarised in Figure 6. At post-operative week 3, there was no significant difference in scores between the groups $(\mathrm{p}=0.1121)$, but at weeks 6, 12 and 24, the total score of the PLG group was significantly higher than that of the control group.

\section{Discussion}

If an osteochondral defect $5 \mathrm{~mm}$ in diameter is made on the femoral condyle of a rabbit and is untreated, it does not heal. By contrast, when such a defect is treated by a PLG scaffold, the cells, which initially attached to the articular surface of the PLG scaffold, proliferate and differentiate into chondrocytes, and the scaffold is absorbed and replaced with newly-formed bone at the deep zone of the defect. We found that at post-operative week 24, regenerated cartilage and a pattern of bone trabeculae at the subchondral zone replicated the appearance of the adjacent normal articular cartilage. Round-shaped chondrocytes at the superficial zone, hypertrophic chondrocytes with a vacuole at the middle, non-calcified and calcified zones and a tidemark between the calcified and the non-calcified zone were detected. It is suggested that the tidemark between the calcified and non-calcified cartilage provides a tethering mechanism for collagen fibrils of the deepest portion of the non-calcified cartilage. ${ }^{9}$ Our results indicate that fullthickness osteochondral defects were repaired and the regenerated articular cartilage and subchondral bone were remodelled, when the osteochondral defect was treated by the PLG scaffold.

In earlier studies, small full-thickness cartilage defects have been shown to fill with blood clots formed by erupted bone-marrow cells including osteoprogenitor cells, and these non-critical-sized cartilage defects healed spontaneously. ${ }^{1,6}$ However, full-thickness osteochondral defects of a critical size have not filled with enough erupted bonemarrow cells and the defect could not be repaired. In our study, consistent with a previous study in a goat model, ${ }^{7}$ untreated osteochondral defects $5 \mathrm{~mm}$ in diameter did not heal. By contrast, when such defects were treated by the PLG scaffold, cells which attached to the scaffold repaired the defect while the scaffold was absorbed. Although it was difficult to identify the origin of the cells which initially attached to the scaffold we speculated that they erupted from the underlying bone marrow. Thereafter, the cells which were attached to the articular surface of the scaffold regenerated cartilage tissue under the influence of synovial fluid and surrounding articular cartilage. Those which were attached to the deep zone of the scaffold regenerated to bone during absorption of the scaffold. We speculate that the porous PLG scaffold plays an important role in trapping erupted bone-marrow cells in the osteochondral defect, leading to regeneration of cartilage and bone while the PLG is absorbed.

Many biomaterials, such as hydroxyapatite, tricalcium phosphate cement, hyaluronic acid, collagen and synthetic polymer have been developed as scaffolds for bone. ${ }^{10}$ Hyaluronan- and polyester-based scaffolds ${ }^{11}$ or a composite of porous hydroxyapatite, synthetic polymer and bone morphogenetic protein (BMP)- $2^{12}$ promote repair of fullthickness cartilage defects. The porosity, rate of degradation and mechanical properties of the materials play a critical role in the regeneration of bone and cartilage by using 


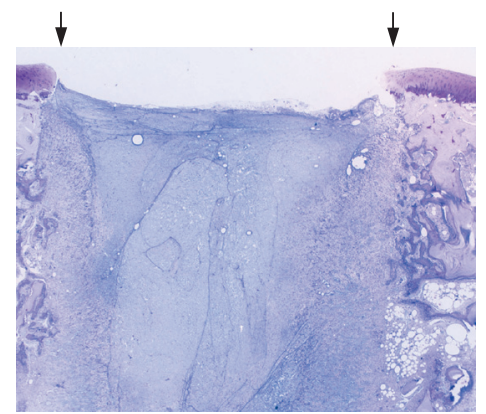

Fig. 5a

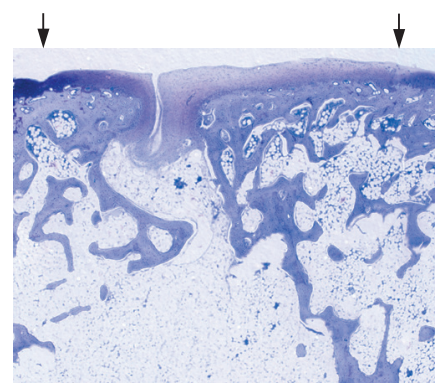

Fig. $5 d$

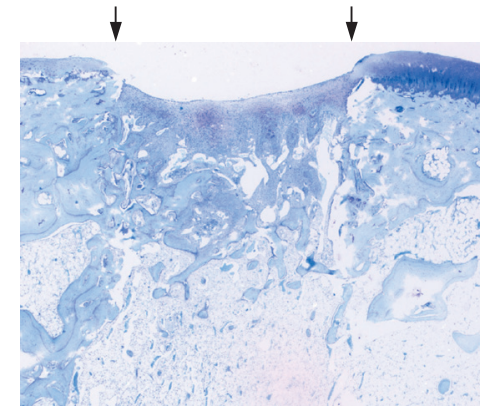

Fig. $5 b$

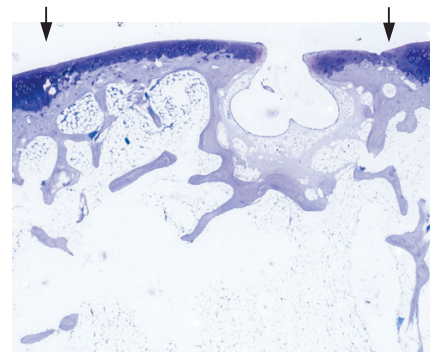

Fig. $5 \mathrm{e}$

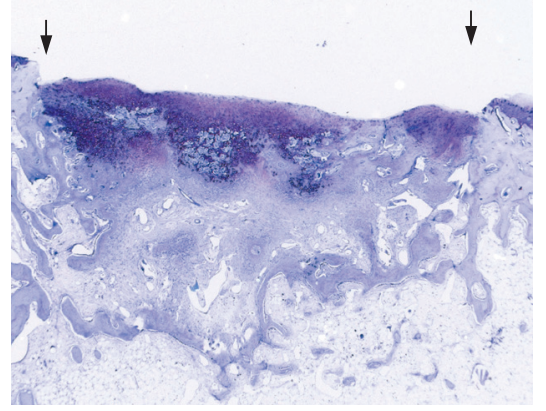

Fig. 5c

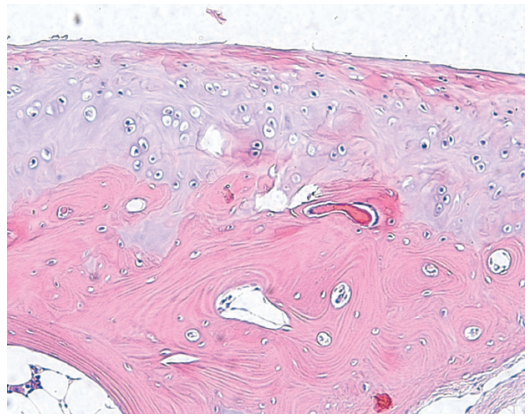

Fig. $5 f$

Photomicrograph showing the histological findings in the control group. Histological findings stained with toluidine blue (a, b, $c$, $d, e)$ and with haematoxylin and eosin (f). The original magnification is $\times 25(a, b, c, d, e)$ and $\times 200$ (f).

Table II. Histological scores for the poly (DL-lactide-co-glycolide) (PLG) and control groups with SD (range)

\begin{tabular}{|c|c|c|c|c|c|c|}
\hline \multirow{2}{*}{$\begin{array}{l}\text { Time after surgery } \\
\text { (wks) }\end{array}$} & \multicolumn{5}{|c|}{ Evaluation category } & \multirow[b]{2}{*}{ Total score } \\
\hline & A & B & C & D & E & \\
\hline \multicolumn{7}{|l|}{$\overline{P L G}$} \\
\hline 3 & $1.3 \pm 0.5(1$ to 2$)$ & $1.0 \pm 0(1)$ & $0.3 \pm 0.5(0$ to 1$)$ & $0 \pm 0(0)$ & $0 \pm 0(0)$ & $2.7 \pm 0.8(2$ to 4$)$ \\
\hline 6 & $2.2 \pm 0.8(1$ to 3$)$ & $1.7 \pm 0.5(1$ to 2$)$ & $1.7 \pm 0.5(1$ to 2$)$ & $0.8 \pm 0.4(0$ to 1$)$ & $0.7 \pm 0.5(0$ to 1$)$ & $7.0 \pm 2.1(4$ to 9$)$ \\
\hline 12 & $2.5 \pm 0.5(2$ to 3$)$ & $2.0 \pm 0.6(1$ to 3$)$ & $2.0 \pm 0.6$ (1 to 3$)$ & $1.5 \pm 0.5(0$ to 2$)$ & $1.0 \pm 0(1)$ & $9.0 \pm 2.0$ (7 to 12$)$ \\
\hline \multicolumn{7}{|l|}{ Control } \\
\hline 3 & $1.0 \pm 0.9(0$ to 2$)$ & $0.7 \pm 0.5(0$ to 1$)$ & $0.2 \pm 0.4$ ( 0 to 1$)$ & $0 \pm 0(0$ to 1$)$ & $0 \pm 0(0)$ & $1.8 \pm 1.6(0$ to 4$)$ \\
\hline 6 & $1.0 \pm 0.9(0$ to 2$)$ & $0.8 \pm 0.8$ (0 to 2$)$ & $0.5 \pm 0.5(0$ to 1$)$ & $0.1 \pm 0.4(0$ to 1$)$ & $0.1 \pm 0.4(0$ to 1$)$ & $2.7 \pm 2.3$ ( 0 to 6$)$ \\
\hline 12 & $1.3 \pm 0.8(0$ to 2$)$ & $0.8 \pm 0.4(0$ to 1$)$ & $1.0 \pm 0.9(0$ to 2$)$ & $0 \pm 0(0)$ & $0 \pm 0(0)$ & $3.2 \pm 1.5(1$ to 5$)$ \\
\hline 24 & $1.7 \pm 0.5(1$ to 2$)$ & $1.2 \pm 0.4(1$ to 2$)$ & $1.0 \pm 0.6(0$ to 2$)$ & $0.2 \pm 0.4(0$ to 1$)$ & $0 \pm 0(0)$ & $3.7 \pm 1.0(2$ to 5$)$ \\
\hline
\end{tabular}

the biomaterial scaffolds. ${ }^{10,11}$ Lower porosity stimulates osteogenesis by suppressing cell proliferation and forcing cell aggregation in vitro. Higher porosity and pore size result in greater ingrowth of bone to the scaffold, but diminish the mechanical properties in vivo. Therefore, a balance of the porosity and pore size, and the rate of remodelling and of degradation of the scaffold are important for biomaterial scaffolds.

Polylactic acid, PLG, and polypropylene fumarate have been used as synthetic polymers in the reconstruction of bone and other tissues. ${ }^{10}$ Although highly vascular regions such as the craniofacial skeleton appear to tolerate absorb- able fixation, ${ }^{13}$ an inflammatory response is induced by implants of biodegradable synthetic polymer used in regions of poor vascularity. ${ }^{14}$ The poly (L-lactic acid) (PLLA)/poly(glycolic acid) (PGA) copolymer has a degradation time which lies between that of PGA and PLLA and thus the implantation of a PLLA/PGA copolymer avoids the inflammatory complications related to the rapid degradation of PGA, and decreases the complications related to the prolonged retention of hardware associated with PLLA implants. ${ }^{15}$

Features of synthetic polymer scaffolds, such as form, porosity, pore size, rate of degradation and mechanical 


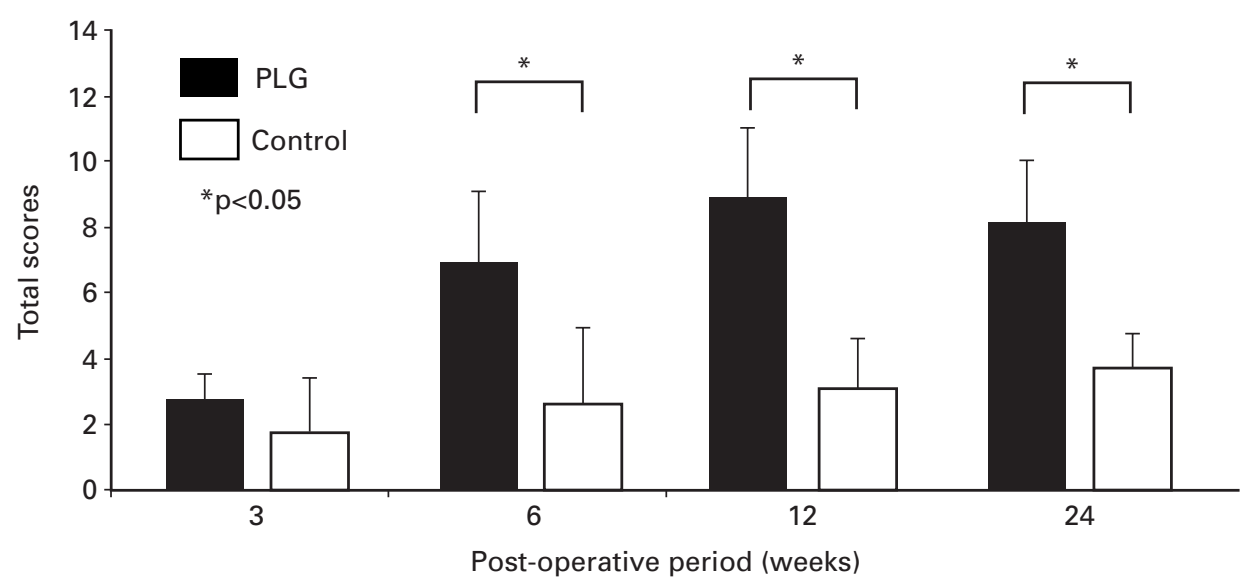

Fig. 6

Bar chart showing that the total score of the PLG (poly (DL-lactide-co-gylcolide) group was significantly higher than that of the control group at post-operative weeks 6,12 and 24 .

properties differ between the types of polymer and their method of fabrication. ${ }^{10}$ The fabrication techniques of synthetic polymers include salt-leaching, gas foaming, electrospinning, and freeze-drying. In our study, in order to repair the osteochondral defect, we developed a new scaffold made from PLG using a freeze-drying technique. The pore size of the PLG scaffold was less than $100 \mu \mathrm{m}$ and its mean total porosity was 0.83 . Although the pore size and the porosity of the scaffold used in our study may not be ideal for the regeneration of cartilage and bone in man, we believe that the balance of porosity and pore size and rate of remodelling and degradation of the PLG scaffold was suitable for full-thickness osteochondral defects in a rabbit model.

Several cytokines, BMP-2, BMP-7, and fibroblast growth factor- 2 have been suggested to have application in the repair of articular cartilage. ${ }^{12,16,17}$ The use of a composite of scaffolds with cytokines and cultured cells for repairing defects of articular cartilage has been reported. ${ }^{11,12,18}$ Our study suggests that the new PLG scaffold has the advantage of reducing the side-effects due to cytokines and growth factors. However, the synthetic polymer scaffold implanted in the osteochondral defect may inhibit cell migration from the bone marrow and surrounding cartilage to the defect while the scaffold is absorbed. Further investigation will be needed to determine the optimal pore size, porosity and fabricating technique required before repair of osteochondral defects by the PLG scaffold can be used in clinical applications.

We thank C. Satoh, H. Kaneko, and E. Kitazono for producing the PLG scaffold and M. Nagata for assistance with the histological experiments. This study was partially supported by Scientific Research Grant of Kobe University Graduate School of Medicine in 2004 and Scientific Research Grant of Shinryokukai in 2004

No benefits in any form have been received or will be received from a commercial party related directly or indirectly to the subject of this article.

\section{References}

1. Caplan Al, Elyaderani M, Mochizuki Y, Wakitani S, Goldberg VM. Principles of cartilage repair and regeneration. Clin Orthop 1997;342:254-69.

2. Menche DS, Vangsness CT Jr, Pitman M, Gross AE, Peterson L. The treatment of isolated articular cartilage lesions in the young individual. In: Cannon WD Jr, ed. AAOS Instructional Course Lectures. 1998:47:505-15.

3. Johnson LL. Clinical methods of cartilage repair: arthroscopic abrasion arthroplasty: a review. Clin Orthop 2001;391(Suppl):306-17.

4. Terukina M, Fujioka H, Yoshiya S, et al. Analysis of the thickness and curvature of articular cartilage of the femoral condyle. Arthroscopy 2003;19:969-73.

5. Brittberg M, Lindahl A, Nilsson A, et al. Treatment of deep cartilage defects in the knee with autologous chondrocyte transplantation. N Eng/ J Med 1994;331:889-95.

6. Shapiro F, Koide S, Glimcher MJ. Cell origin and differentiation in the repair of full-thickness defects of articular cartilage. J Bone Joint Surg [Am] 1993;75-A:532-53.

7. Jackson DW, Lalor PA, Aberman HM, Simon TM. Spontaneous repair of fullthickness defects of articular cartilage in a goat model: a preliminary study. J Bone Joint Surg [Am] 2001;83-A:53-64.

8. Wakitani S, Goto T, Pineda SJ, et al. Mesenchymal cell-based repair of large, fullthickness defects of articular cartilage. J Bone Joint Surg [Am] 1994;76-A:579-92.

9. Redler I, Mow VC, Zimmy ML, Mansell J. The ultrastructure and biomechanical significance of the tidemark of articular cartilage. Clin Orthop 1975;112:357-62.

10. Karegeorgiou V, Kaplan D. Porosity of $3 \mathrm{D}$ biomaterial scaffolds and osteogenesis. Biomaterials 2005;26:5474-91.

11. Solchaga LA, Temenoff JS, Gao J, et al. Repair of osteochondral defects with hyaluronan- and polyester-based scaffolds. Osteoarthritis Cartilage 2005;13:297-309.

12. Tamai N, Myoui A, Hirao $\mathbf{M}$, et al. A new biotechnology for articular cartilage repair: subchondral implantation of a composite of interconnected porous hydroxyapatite, synthetic polymer (PLA-PEG), and bone morphogenetic protein-2 (rhBMP-2). Osteoarthritis Cartilage 2005; 13:405-17.

13. Edwards RC, Kiely KD, Eppley BL. The fate of resorbable poly-L-lactic/polyglycolic acid (LactoSorb) bone fixation devices in orthognathic surgery. J Oral Maxillofac Surg 2001;59:19-25

14. Böstman O, Hirvensalo E, Mäkinen J, Rokkanen P. Foreign-body reactions to fracture fixation implants of biodegradable synthetic polymers. J Bone Joint Surg [Br] 1990;72-B:592-6.

15. Larsen MW, Pietrzak WS, DeLee JC. Fixation of osteochondritis dissecans lesions using poly (I-lactic acid)/poly (glycolic acid) copolymer bioabsorbable screws. Am J Sports Med 2005;33:68-76.

16. Hidaka C, Goodrich LR, Chen CT, et al. Acceleration of cartilage repair by genetically modified chondrocytes over expressing bone morphogenetic protein-7. J Orthop Res 2003;21:573-83.

17. Kaul G, Cucchiarini M, Arntzen D, et al. Local stimulation of articular cartilage repair by transplantation of encapsulated chondrocytes overexpressing human fibroblast growth factor 2 (FGF-2) in vivo. J Gene Med 2006;8:100-11.

18. Ito $\mathbf{Y}, \mathbf{O c h i} \mathbf{M}$, Adachi $\mathbf{N}$, et al. Repair of osteochondral defect with tissue-engineered chondral plug in a rabbit model. Arthroscopy 2005;21:1155-63. 